\title{
IGNITE: a tool for adaptive fire management and biodiversity conservation in the Cerrado
}

\author{
Eugênia K.L. Batistal ${ }^{l}$, Geraldo W. Fernandes ${ }^{l}$, José E.C. Figueira $^{l}$ \\ ${ }^{1}$ Universidade Federal de Minas Gerais (UFMG), Belo Horizonte, Brasil \\ * e-mail: biogenia.k@gmail.com
}

\begin{abstract}
Fire has shaped plant evolution and biogeochemical cycles for millions of years in savanna ecosystems. Despite this, fire suppression practices are still prevalent in the Brazilian savannas and generally result in fire regimes incompatible with biodiversity conservation goals. Recently, managers and researchers have discussed and implemented fire management alternatives including more adaptive and integrated approaches. In adaptive management, decisions are made as part of an ongoing process of review and evaluation of results to improve understanding of system responses to implemented practices and adjust them if necessary. Implementing an adaptive approach into fire management requires the ability to deal with uncertainty, consider multiple management options at the same time, overcome institutional barriers, apply innovative monitoring approaches, resolve management conflicts and improve the poor interaction between scientists, managers and local people. Of course, fire management practices raise relevant issues such as: What spatial and temporal patterns of fire are needed to sustain ecosystem conservation values and biodiversity, and how can such regimes be properly implemented and monitored? To answer these questions and assist managers in decision making process, we are developing a management software which is called IGNITE, a free tool, planned and structured specifically for fire management in the Brazilian Cerrado. The IGNITE should lead managers through three modules: fire management planning, execution and monitoring. At each step, users should add qualitative information and enter spatial data such as the land cover mapping and areas affected by wildfires and prescribed burns in previous years. The software aims to identify areas susceptible to inappropriate fire regimes, helping to guide management decisions. In addition, by proposing targets, indicators and protocols for research and monitoring we hope to consolidate the adaptive fire management and encourage managers and researchers to engage more deeply in the process. The IGNITE has the potential to reduce scientific uncertainties and enable, in medium and long term, the building of a database for biodiversity conservation in the Cerrado.
\end{abstract}

Keywords: adaptive fire management, management software, Cerrado 